\title{
Prognostic Significance of Hormone Receptor (ER/PR) Status Inendometrial Carcinoma in Black Women: Implications with Lymph Node Metastasis

\author{
Manan Shah ${ }^{1}$, Gabriela Oprea ${ }^{2}$, Saba Shafi ${ }^{3}$, Phani Keerthi Surapaneni ${ }^{1}$, Sandeep \\ Sasidharan ${ }^{4}$, and Sanjay Jain ${ }^{1}$
}

${ }^{1}$ Department of Internal Medicine and Hematology-Oncology, Morehouse School of Medicine, Atlanta, Georgia, USA

${ }^{2}$ Department of Pathology, Emory University School of Medicine, Atlanta, Georgia, USA

${ }^{3}$ Department of Pathology, Yale University School of Medicine, New Haven, Connecticut, USA

${ }^{4}$ Statistician, Des Moines, lowa city, lowa, USA

*Corresponding author: Manan Shah, Department of Internal Medicine and Hematology-Oncology, Morehouse School of Medicine, Atlanta, Georgia, USA

\begin{abstract}
Loss of estrogen and progesterone receptors (ER/ PR) independently predicts lymph node metastasis and poor prognosis. Black females in comparison to their white counterparts are diagnosed at a later stage, with higher grade, poorer prognostic histology, and worse outcomes. We reviewed black females $(n=70)$ diagnosed with endometrial cancer at our institution from 2015-2018 including endometroid/ non-endometroid histology, FIGO stage I-IVA, grade 1-3 for $E R / P R$ receptors and presence or absence of pelvic lymph nodes based on preoperative endometrial curettage and post-surgical pathology specimens. Majority of our patients were postmenopausal (59 out of 70 ) $84 \%$. FIGO Stage 1 and 2 constituted $72 \%$ (50 out of 70 ). Endometroid histology constituted 39 out of 70 (55\%) cases and non-endometroid histology which includes serous, clear cell and carcinosarcomas constituted $45 \%$ of the cases. Loss of estrogen receptor (ER-) was observed in 19 of 70 patients $(27 \%)$ and loss of progesterone receptor (PR-) was seen in 22 patients (31\%) which were significantly associated with lymph node metastasis ( $p$ value <0.05). Subgroup analyses showed a significant association between PR+ and absence of lymph node metastasis in cases of endometrioid cancer ( $p$ value $<0.05$ ). Nevertheless, there was no statistically significant association ( $P$ value .10) between ER receptors and lymph node metastasis in patients with endometrioid cancer. In non-endometrioid histology both ER- and PR- were found to have statistically significant lymph node involvement. This study aimed to investigate if the correlation between hormone receptor status and lymph node metastasis holds true in black females which could aid in treatment strategies.
\end{abstract}

\section{Introduction}

Endometrial cancer is the most common gynecological malignancy in the United States [1]. Endometrial cancers are further divided into endometroid and non-endometroid histology. Non-endometroid histology includes serous, carcinosarcomas which are associated with poorer prognosis. Risk factors for endometrial cancers mainly includes obesity, early menarche and late menopause, unopposed estrogen, and family history of endometrial cancer [2]. About $80 \%$ of the endometrial cancers are endometroid and are diagnosed early with better prognosis [3]. There is a subset of the population that fail to reach 5 year overall survival even with endometroid histology and early stage of the disease [4]. Various prognostic indicators including FIGO stage, grade and histology have been used for risk assessment and for treatment decisions. However, estrogen (ER) and progesterone (PR) receptor status are considered the most significant prognostic markers for endometrial cancer [5-7].

Racial disparity especially in the survival rates for endometrial cancer is evident and has been increasing over the last 10 years [8]. Black women have $90 \%$ higher 5 -year mortality than white females. Black women are diagnosed at an advanced stage and have a higher

Citation: Shah M, Oprea G, Shafi S, Surapaneni PK, Sasidharan S, et al. (2021) Prognostic Significance of Hormone Receptor (ER/PR) Status Inendometrial Carcinoma in Black Women: Implications with Lymph Node Metastasis. Int J Cancer Clin Res 8:150. doi.org/10.23937/2378-3419/1410150

Accepted: April 17, 2021: Published: April 19, 2021

Copyright: (c) 2021 Shah M, et al. This is an open-access article distributed under the terms of the Creative Commons Attribution License, which permits unrestricted use, distribution, and reproduction in any medium, provided the original author and source are credited. 
proportion of non-endometroid histology associated with poor prognosis [9].

To address the gaps between black and white women comprehensive studies are required to assist us in better treatment decisions to improve survival in black women. Our study aimed to investigate if the correlation between hormone receptor status and lymph node metastasis holds true in black females which could aid in treatment protocols.

Gene expression of ER and PR receptors have been directly associated with prognosis of endometrial cancer in terms of survival. Loss of ER- and PR- receptors independently predicts lymph node metastasis and shorter disease-free survival (DFS) [10]. Pre-operative endometrial curettage or biopsy is the first step in diagnosis and to identify receptor (ER/PR) status. Identifying the receptor status especially in surgical operable stages can help to individualize surgical therapy in endometrial cancer. Routine lymph node dissection has not been confirmed to improve survival and is associated with increased complications [11]. Hence, receptor status could aid us in moving forward with aggressive treatment with pelvic lymphadenectomy especially in our population of black females. Our aim is to help in creating an algorithm for management of endometrial cancer for our population.

Integrating genomic biomarkers like ER/PR receptors, p53, Her-2, MMR panel with clinical and pathological parameters will improve risk stratification and will guide us in predicting the future course of treatment and possible long-term survival. Unlike some cancers like breast, where genomic biomarkers have been incorporated for tailored therapy, targeted agents are still in its primitive stage in endometrial cancers [10].

Our study aimed at investigating 1) association of loss of ER and PR receptors with lymph node metastasis as detected in the post-operative specimens; 2 ) role of $E R$ and $P R$ receptors in both endometroid and non-endometroid subtypes separately; 3 ) to check if receptor hypothesis holds true for black females; 4) subgroup analysis to identify genomic markers for non-endometroid variants.

\section{Methods and Materials}

\section{Participants}

We reviewed black females with endometrial cancer ( $n$-70) at our institution diagnosed between 2015-2018. Patients included in our study underwent pre-operative endometrial curettage or biopsy followed by trans-abdominal or laparoscopic surgery with lymph node dissection/sampling or debulking surgery in advanced stage cancer. We included patients with FIGO stage 1-IVA, Grade 1-3, both endometrioid and non-endometrioid histology. Patients with distant metastasis were excluded from the study. Baseline characteristics including age, BMI, pre/post-menopausal status and were noted. Genomic biomarkers specially ER and PR receptors status were noted for both endometroid/non-endometroid histology. Pelvic and para-aortic nodes involvement was identified from post-operative specimens. A subgroup analysis for identifying genomic targets in the non-endometroid subtype was performed. The potential targets identified were p16, p53, MMR panel and Her- 2 in addition to the ER/PR status.

\section{Immunohistochemical staining and evaluation}

ER,PR and p53 expression was assessed by IHC's using formalin-based paraffin embedded samples. Molecular targets including p16, Her-2 and MMR panel were identified on tissue samples. The expression of ER, PR and p53 status were evaluated by at least 2 pathologists. Staining of ER/PR was considered positive if the nuclei were stained in $>1 \%$ of the cells. The status of p53 was graded as "p53 loss", < 1\% was stained, "p53 normal", $1 \%-50 \%$ stained and over-expression if $>50 \%$ strongly stained.

\section{Statistical analysis}

Statistical analyses were performed with Python 3.7 (SciPy, NumPy, Pandas, and Matplotlib libraries) using Fisher's Exact test exploring associations between categorical variables (ER/PR and Lymph nodes Metastasis). To corroborate the findings, estimated odds ratios (OR) for lymph node metastasis to ER/PR was calculated. The analysis was done for independent ER/ PR positive and negative, for all subjects and for the two subtypes (endometrioid and non-endometrioid). All statistical tests were two-sided and considered significant if $P<0.05$. Post-hoc power calculation was done using the software $\mathrm{G}^{*}$ Power 3.1

Descriptive statistics were used to characterize baseline parameters like age, BMI including molecular targets for non-endometroid histology. Weighted percentages were used to summarize the data. Our study was approved by the institutional review board (IRB).

\section{Results}

\section{General patient characteristics}

We included all black females only ( $n-70)$ that were diagnosed with endometrial cancer from 2015-2018 and who underwent laparoscopic or transabdominal surgery or debulking surgery (Table 1).

- Age: The mean age of our population was 61 years with a range from $33-80$ years.

- Body Mass Index: The mean BMI of our patients were $36.94 \mathrm{~kg} / \mathrm{m}^{2}$.

- Menstrual status: Majority of our patients 59/70 (84\%) were post-menopausal while remaining $16 \%$ were pre-menopausal.

- Stage: Majority of our patients 50/70 (71\%) were 
Table 1: Characteristics of 70 endometrial cancer patients included in the study.

\begin{tabular}{|c|c|c|}
\hline Characteristics & $N$ & $\%$ \\
\hline Mean age (years) & 61.45 & \\
\hline Range & 33-94 & \\
\hline \multicolumn{3}{|l|}{ Menopausal status } \\
\hline Premenopausal & 59 & 84.28 \\
\hline Postmenopausal & 11 & 15.71 \\
\hline \multicolumn{3}{|l|}{ FIGO stage } \\
\hline I & 45 & 64.28 \\
\hline II & 5 & 7.14 \\
\hline III & 10 & 14.28 \\
\hline IV & 10 & 14.28 \\
\hline \multicolumn{3}{|l|}{ Histological subtype } \\
\hline Endometrioid & 39 & 55.71 \\
\hline Serous & 19 & 27.14 \\
\hline Carcinosarcoma & 12 & 17.14 \\
\hline \multicolumn{3}{|c|}{ Histological differentiation } \\
\hline Grade 1 & 23 & 32.85 \\
\hline Grade 2 & 15 & 21.42 \\
\hline Grade 3 & 32 & 45.71 \\
\hline \multicolumn{3}{|c|}{ Estrogen receptor status } \\
\hline Yes & 51 & 72.85 \\
\hline No & 19 & 27.14 \\
\hline \multicolumn{3}{|c|}{ Progesterone receptor status } \\
\hline Positive & 48 & 68.57 \\
\hline Negative & 22 & 31.42 \\
\hline \multicolumn{3}{|c|}{ Pelvic lymph node sampling } \\
\hline Yes & 20 & 28.57 \\
\hline No & 50 & 71.42 \\
\hline \multicolumn{3}{|c|}{ Status at 1-year follow-up } \\
\hline Alive & 68 & 97.14 \\
\hline Dead & 2 & 2.85 \\
\hline \multicolumn{3}{|c|}{ Status at time of follow-up } \\
\hline Stable & 42 & 60 \\
\hline Progressive & 28 & 40 \\
\hline
\end{tabular}

diagnosed with FIGO stage I and Stage II disease while the remaining $28 \%$ were diagnosed with FIGO stage III and IVA. Patients with distant metastasis were excluded from the study.

- Grade: Majority of our patients 32/70 (45\%) had grade 3 disease followed by grade 1 in 32\%.

- Histology: Endometroid cancers formed 39/70 (55\%) of our sample followed by non-endometroid which includes serous in $22 \%$ and carcinosarcoma in $16 \%$. (Figure 1).

\section{Hormone receptors and lymph nodes}

We observed presence of hormone receptors which involved ER and PR receptors in pre-operative endometrial specimens using biopsy or curettage samples.
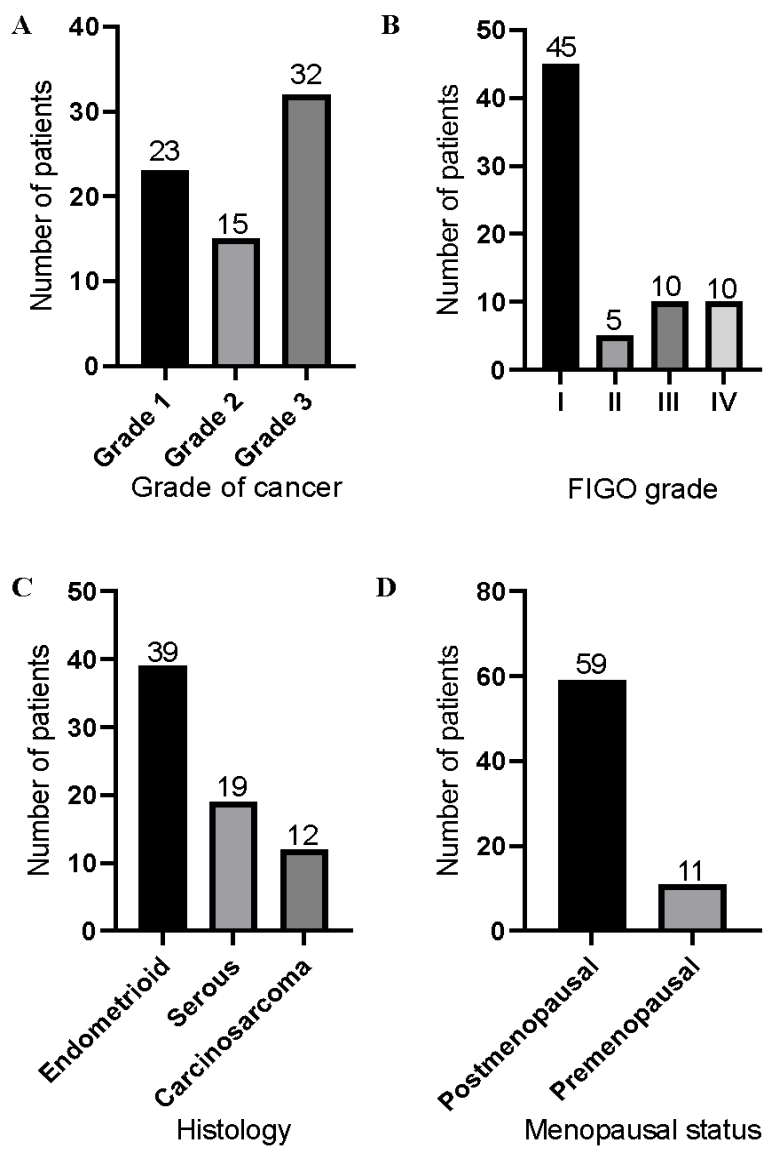

Figure 1: Patient Demographic data. Bar diagrams showing A) Grade of cancer; B) FIGO stage; C) Histology; D) Menopausal status in the represented patient population.

Presence or absence of lymph node involvement was detected in samples obtained through laparoscopic or transabdominal or debulking surgery. Presence or absence of hormone receptors and its correlation with lymph node metastasis was observed in the entire sample and a subgroup analysis was done separately for endometroid and non-endometroid histology. Estrogen receptors were positive $(E R+)$ in $51 / 70(72 \%)$ of our samples and $P R$ receptors were positive $(\mathrm{PR}+)$ were positive in $48 / 70$ (68\%). On subgroup analysis in endometroid cancers 35/39 (89\%) were ER+ while 36/39(92\%) were $\mathrm{PR}+$. The non-endometroid variant showed $\mathrm{ER}+$ in 16/31 $(51 \%)$ and $P R+12 / 31(38 \%)$ of the samples (Figure 2).

Loss of estrogen receptor (ER-) was observed in 19 of 70 patient samples $(27 \%)$ and found to be significantly associated with lymph node metastasis $(p<0.05)$. Progesterone receptor loss was noted (PR-) in $22(31 \%)$ and was associated with metastasis of lymph node (Table 2 ). On sub- group analysis (Table 3 and Table 4) the association between ER/PR with lymph node metastasis in patients with endometrioid and non- endometrioid (comprising of serous, carcinosarcoma and mixed) endometrial cancer showed significant association between PR+ and absence of lymph node metastasis in endometrioid cancer type. There was no statistically significant association ( $P$ value .10 ) between ER and lymph node me- 
A
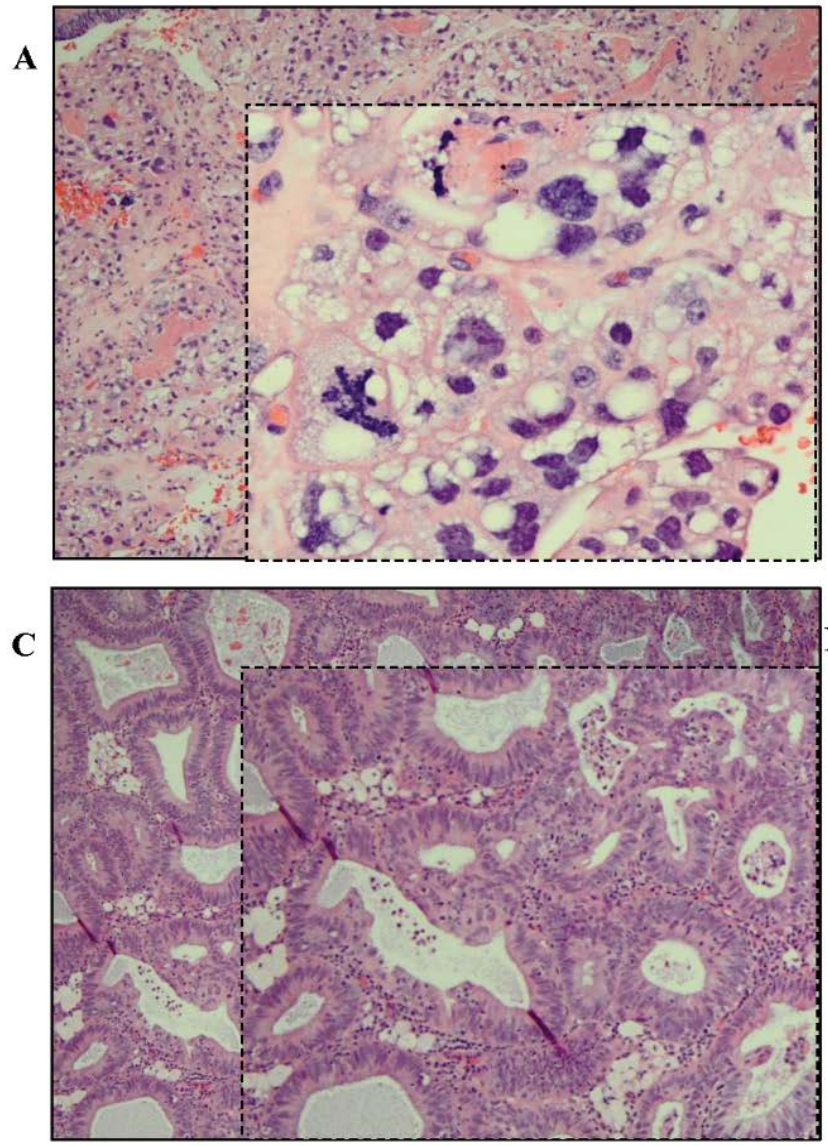
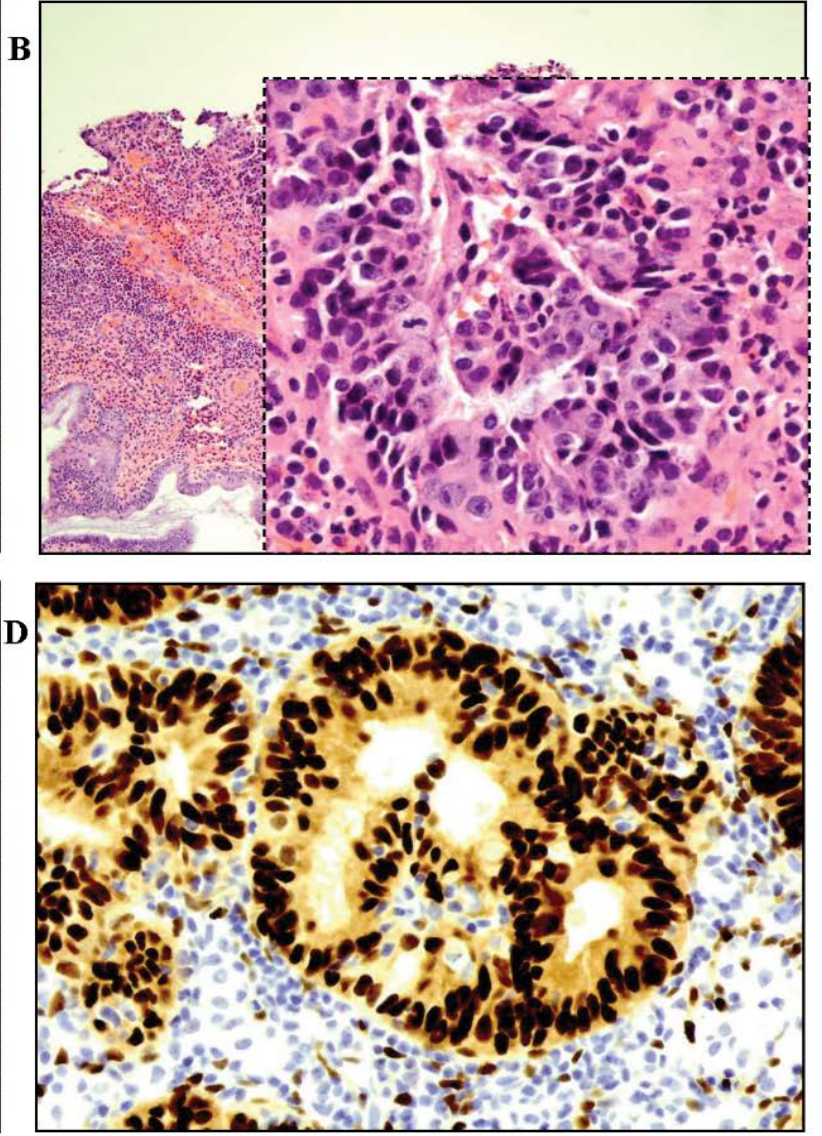

Figure 2: Photomicrographs of endometrial samples. A) H\&E of clear cell endometrial carcinoma (at magnification 20x, inset: 40x); B) H\&E image of endometrioid carcinoma, FIGO grade II (at magnification 20x, inset: 40x); C) H\&E image of endometrioid carcinoma, FIGO grade I (at magnification 20x, inset: 40x); D) ER positivity seen as strong nuclear staining in grade I endometrioid carcinoma.

Table 2: Lymph node sampling in 70 endometrial cancer patients and correlation with receptor biomarkers evaluated by Fisher's Exact test.

\begin{tabular}{|l|l|l|l|}
\hline Variables & $\begin{array}{l}\text { Lymph node metastasis } \\
\text { No }\end{array}$ & $\begin{array}{l}\text { Lymph node metastasis } \\
\text { Yes } \\
\text { N (\%) }\end{array}$ & -value \\
\hline ER positive & $45(88)$ & $6(12)$ & $1.35 \mathrm{e}^{-06}$ \\
\hline ER negative & $5(26)$ & $14(74)$ & $1.35 \mathrm{e}^{-06}$ \\
\hline PR positive & $45(94)$ & $3(6)$ & $2.86 \mathrm{e}^{-09}$ \\
\hline PR negative & $5(23)$ & $17(77)$ & $2.86 \mathrm{e}^{-09}$ \\
\hline
\end{tabular}

Table 3: Lymph node sampling in 39 endometrioid subtype patients and correlation with receptor biomarkers evaluated by Fisher's Exact test.

\begin{tabular}{|l|l|l|l|}
\hline Variables & Lymph node metastasis & $\begin{array}{l}\text { Lymph node metastasis } \\
\text { Yes }\end{array}$ & P-value \\
\hline No & N (\%) & \\
\hline ER positive & $31(89)$ & $4(11)$ & 0.10 \\
\hline ER negative & $2(50)$ & $2(50)$ & 0.10 \\
\hline PR positive & $33(92)$ & $3(8)$ & 0.002 \\
\hline PR negative & $0(0)$ & $3(100)$ & 0.002 \\
\hline
\end{tabular}


Table 4: Lymph node sampling in 31 non- endometrioid subtype patients and correlation with receptor biomarkers evaluated by Fisher's Exact test.

\begin{tabular}{|l|l|l|l|}
\hline Variables & Lymph node metastasis & Lymph node metastasis & P-value \\
& $\begin{array}{l}\text { No } \\
\text { N (\%) }\end{array}$ & $\begin{array}{l}\text { Yes } \\
\text { N (\%) }\end{array}$ & \\
\hline ER positive & $14(88)$ & $2(12)$ & 0.00024 \\
\hline ER negative & $3(20)$ & $12(80)$ & 0.00024 \\
\hline PR positive & $12(100)$ & $0(0)$ & $8.83 e^{-05}$ \\
\hline PR negative & $5(26)$ & $14(74)$ & $8.83 \mathrm{e}^{-05}$ \\
\hline
\end{tabular}

tastasis in patients with endometrioid type cancer. In case of non-endometrioid (comprising of serous, carcinosarcoma and clear cell) both ER- and PR- were found to be significant with respect to metastasis to the lymph nodes $(p<0.05)$.

\section{Potential genomic targets in non-endometroid cancers}

A total of 31 patients were diagnosed with non-endometroid cancers which included serous, carcinosarcomas subtypes and are rare variants commonly seen in black females in contrast to their white counterparts. We conducted a sub-analysis to identify potential molecular targets which could be incorporated in future for the management of these variants which in general have poor prognosis and survival rates. Molecular markers included hormone receptor status (ER/PR), p16, p53, Her-2/neu, and the MMR gene panel (MLH1, PMS2, MSH2, MSH6). Molecular studies revealed ER+ in $41 \%$ and $P R+$ in $33 \%$ with carcinosarcomas and $43 \%$ and $31 \%$ with serous subtypes respectively. All patients with carcinosarcomas were Her-2/neu negative while $25 \%$ were positive in serous tumors. $88 \%$ cases were strongly positive for both p53 and p16 in the serous variant. p53 and p16 were negative in carcinosarcomas. All patients showed an intact MMR gene panel.

All our patients were closely followed up as an outpatient. Patients eligible for adjuvant therapy post-surgery received necessary chemo-radiation according to the guidelines. At the end of one-year post-treatment $68 / 70$ (97\%) were alive. However, 60\% patients had stable disease and nearly $40 \%$ had progression in the form of metastasis to lung, vagina, or local spread. A noticeable observation made was majority of our patients with progression at one year follow-up post treatment was from the non-endometroid group.

\section{Discussion}

We report for the first time the role of hormonal receptors and its significance with respect to lymph node metastasis in black women. Involvement of pelvic and para-aortic lymph nodes by itself is an indicator of poor outcomes in addition to race which is associated with poor survival. Our study aimed to observe if hormone receptor status and its association with lymph node metastasis holds true in black women which could help in creating proper algorithms for aggressive treatment in this vulnerable group [9]. This would also help us in identifying individuals who would benefit from pelvic lymph node dissection which has its own complications [11]. Black women have been seen to be diagnosed at a later stage and have higher proportion of non-endometroid histology [12,13].

Lymph node sampling is considered an important component in endometrial cancer management in particular for high grade and high-risk endometrial cancer [14]. Routine lymphadenectomy in every patient with endometrial cancer is not recommended, as it is associated with longer operative times and significant post-operative complications especially in obese women which form a significant proportion of black women diagnosed with endometrial cancer [15]. Our study reported a mean BMI of $36.94 \mathrm{~kg} / \mathrm{m}^{2}$ keeping these patients at a higher risk of complications if subjected to extensive pelvic and para-aortic lymph node dissection. Improved tools to identify prognostic indicators like hormone receptors status (ER/PR) can guide us in identifying patients who will benefit from lymph node dissection. Pre-operative curettage or biopsy samples for receptor status identification should form the first step in the algorithm for management of endometrial cancers. Studies have also shown that non-endometroid subtypes which are higher in black women and associated with poor outcomes will benefit from these tools to a greater extent. Non-endometroid cancers have been associated with deeper myometrial infiltration and have consistently demonstrated lymph node metastasis [16]. Larger and adequately powered randomized trials are required to study the effects of planned lymphadenectomy in black women who satisfy the pre-requisite based on hormone receptors which is beyond the scope of this study.

Various studies identifying the prognostic value of ER and PR receptors were evaluated across various histological subtypes $[17,18]$. These studies have independently predicted prognostic value of ER and PR receptors in early stage and low-grade endometrial cancer. A study conducted by Trovik, et al. significantly predicted loss or ER (-) and PR (-) and its association with lymph node involvement thereby adding predictive and prognostic value specially in the low-risk group. This study also pre- 
dicted poor disease-free survival (DFS) in patients with a negative receptor status [18]. In our study, which is probably the first study to test this hypothesis in black women, who generally have a higher mortality rate and late stage of presentation in comparison to their white counterparts. We reported a statistically significant association of loss of ER (-) and PR (-) with positive lymph node involvement. On subgroup analysis with endometroid and non-endometroid subtypes we observed significant predictive value of both ER and PR receptors independently specially in serous and carcinosarcomas. This information can be utilized while addressing treatment strategies for black women with endometrial cancer. We strongly recommend conducting larger multicentric trials on black women for better understanding of the disease pattern and course of disease in black females. Creating a treatment algorithm that could be acceptable universally for this group could help in improving survival outcomes.

Studies have reported unfavorable subtypes like serous and carcinosarcomas in higher proportions in black women [19]. We reported potential molecular targets in these subtypes which could improve treatment regimes in the future. Our study revealed ER+ in $41 \%$ and $P R+$ in $33 \%$ with carcinosarcomas and $43 \%$ and $31 \%$ with serous variants respectively. All patients with carcinosarcomas were Her-2/neu negative while $25 \%$ were positive in serous tumors. $88 \%$ cases were strongly positive for both $\mathrm{p} 53$ and $\mathrm{p} 16$ in the serous variant. All patients showed an intact MMR gene panel. Similar studies have been conducted which used these targets [20]. Incorporating molecular testing in the treatment algorithm especially in black women with non-endometroid subtype is our recommendation.

Biomarkers like ER/PR and Her-2 status have been incorporated in the treatment algorithm for breast and gastric cancers. Despite sufficient studies in endometrial cancers, these biomarkers are not routinely used to tailor endometrium cancer management. Our study can add to the database and form the basis of a multicentric prospective trial in black women. We recommend using these biomarkers in patient risk stratification and developing targeted agents in a metastatic setting.

Our study was not designated to test the role of lymphadenectomy in endometrial cancer but to incorporate molecular and targeted staging in the treatment algorithm especially in black women. As reported in our data we recommend that hormone status should be incorporated in randomized trials for surgical and systemic treatment. Pre-operative differentiation of patients can also decrease the burden of tertiary centers, as those with local and low-grade disease can be managed at a local center while advanced disease can be referred to specialized centers [21]. This stratification can only be possible if we have well established guidelines and algorithms.

\section{Conclusion}

Molecular staging and targeted agents are the need of the hour in improving survival outcomes in endometrial cancers especially in black women. We recommend large multicentric randomized trials especially from institutes with significant black patient population for widespread acceptance of these recommendations.

\section{References}

1. Kitchener HC, Trimble EL, Endometrial Cancer Working Group of the Gynecologic Cancer Intergroup (2009) Endometrial cancer state of the science meeting. Int J Gynecol Cancer 19: 134-140.

2. Ali AT (2013) Risk factors for endometrial cancer. Ceska Gynekol 78: 448-459.

3. Rose PG (1996) Endometrial carcinoma. N Engl J Med 335: 640-649.

4. Fujimoto $T$, Nanjyo $H$, Fukuda J, Nakamura A, Mizunuma $\mathrm{H}$, et al. (2009) Endometrioid uterine cancer: histopathological risk factors of local and distant recurrence. Gynecol Oncol 112: 342-347.

5. Hertz DL, Henry NL, Kidwell KM, Thomas D, Goddard A, et al. (2016) ESR1 and PGR polymorphisms are associated with estrogen and progesterone receptor expression in breast tumors. Physiol Genomics 2016 48: 688-698.

6. Badve SS, Baehner FL, Gray RP, Childs BH, Maddala T, et al. (2008) Estrogen- and progesterone-receptor status in ECOG 2197: comparison of immunohistochemistry by local and central laboratories and quantitative reverse transcription polymerase chain reaction by central laboratory. J Clin Oncol. 26: 2473-2481.

7. Gong Y, Yan K, Lin F, Anderson K, Sotiriou C, et al. (2007) Determination of oestrogen-receptor status and ERBB2 status of breast carcinoma: a gene-expression profiling study. Lancet Oncol 8: 203-211.

8. Howlader N, Noone AM, Krapcho M, Miller D, Brest A, et al. (2020) SEER Cancer Statistics Review, 1975-2017, National Cancer Institute. Bethesda, MD.

9. Cote ML, Ruterbusch JJ, Olson SH, Lu K, Ali-Fehmi R (2015) The growing burden of endometrial cancer: a major racial disparity affecting black women. Cancer Epidemiol Biomarkers Prev 24: 1407-1415.

10. Salvesen HB, Haldorsen IS, Trovik J (2012) Markers for individualised therapy in endometrial carcinoma. Lancet Oncol 13: e353-e361.

11. Kitchener H, Swart AM, Qian Q, Amos C, Parmar MK (2009) Efficacy of systematic pelvic lymphadenectomy in endometrial cancer (MRC ASTEC trial): a randomised study. Lancet 373: 125-136.

12. Fader AN, Habermann EB, Hanson KT, Lin JF, Grendys EC, et al. (2016) Disparities in treatment and survival forwomen with endometrial cancer: a contemporary national cancer database registry analysis. Gynecol Oncol 143: 98-104.

13. Coates RJ, Click LA, Harlan LC, Robby S, Barett RJ, et al. (1996) Differences between black and white patients with cancer of the uterinecorpus in interval from symptom recognition to initial medical consultation (United States). Cancer Causes Control 7: 328-336.

14. Werner HM, Trovik J, Marcickiewicz J, Tingulstad S, Staff AC, et al. (2013) A discordant histological risk classification in preoperative and operative biopsy in endometrial cancer 
is reflected in metastatic risk and prognosis. Eur $\mathrm{J}$ Cancer 49: 625-632.

15. Panici PB, Basile S, Maneschi F, Lissoni AA, Mauro S, et al. Systematic Pelvic Lymphadenectomy Vs. No Lymphadenectomy in Early-Stage Endometrial Carcinoma: Randomized Clinical Trial. Journal of the National Cancer Institute 100: 1707-1716.

16. Greven KM, Lanciano RM, Corn B, Case D, Randall ME (1993) Pathologic stage III endometrial carcinoma. Prognostic factors and patterns of recurrence. Cancer 71: 36973702.

17. Trovik J, Wik E, Werner HM, Krakstad C, Helland H, et al. (2013) Hormone receptor loss in endometrial carcinoma curettage predicts lymph node metastasis and poor outcome in prospective multicentre trial. Eur J Cancer 49: 34313441.
18. Werner HM, Salvesen HB (2014) Current status of molecular biomarkers in endometrial cancer. Curd Oncol Rep 16: 403.

19. Long B, Liu FW, Bristow RE (2013) Disparities in uterine cancer epidemiology, treatment, and survival among African Americans in the United States. Gynecol Oncol 130: 652-659.

20. de Jong RA, Nijman HW, Wijbrandi TF, Reyners AK, Boezen HM, et al. (2011) Molecular markers and clinical behavior of uterine carcinosarcomas: focus on the epithelial tumor component. Mod Pathol 24: 1368-1379.

21. Chan JK, Sherman AE, Kapp DS, Zhang R, Osann KE, et al. (2011) Influence of gynecologic oncologists on the survival of patients with endometrial cancer. J Clin Oncol 29: 832-838. 